\title{
Corrigendum
}

Tamara van Donge*, Eveline Staub, Andrew Atkinson, Verena Gotta, John van den Anker, Lorenz Risch, Tatjana Welzel and Marc Pfister

\section{Corrigendum to: Age appropriate reference intervals for eight kidney function and injury markers in infants, children and adolescents}

https://doi.org/10.1515/cclm-2021-0232

Corrigendum to: van Donge T, Staub E, Atkinson A, Gotta V, van den Anker J, Risch L, et al. Age appropriate reference intervals for eight kidney function and injury markers in infants, children and adolescents. Clin Chem Lab Med 2021;58(2):373-82. https://doi.org/10.1515/cclm2020-0781.

The authors regret to inform you that it came to our attention that we have discovered an error in our recently published paper "Age appropriate reference intervals for eight kidney function and injury markers in infants, children and adolescents - 2020 Aug 6;59(2):373-382. doi: 10.1515/cclm-2020-0781.”

The paper characterized eight kidney function and injury markers in healthy infants, children and adolescents. The error is related to the medium in which these kidney biomarkers were measured. These biomarkers were in fact measured in plasma and not in serum. Although an incorrect term for the medium is used, we believe that this error does not influence the results and the conclusion of our study as the statistical analysis is not affected by this inaccuracy. In order to be transparent, we would like to have this information printed as an erratum.

*Corresponding author: Tamara van Donge, Pediatric Pharmacology and Pharmacometrics Research, Universitäts-Kinderspital beider Basel (UKBB) Spitalstr. 33, 4031, Basel, Switzerland, Phone: +41 61 70412 12, E-mail: tamara.vandonge@ukbb.ch. https://orcid.org/ 0000-0003-4607-3179

Eveline Staub, Department of Neonatology, Royal North Shore Hospital, St Leonards, Australia Andrew Atkinson, Verena Gotta, Tatjana Welzel and Marc Pfister, Pediatric Pharmacology and Pharmacometrics, University Children's Hospital Basel (UKBB), University of Basel, Basel, Switzerland John van den Anker, Pediatric Pharmacology and Pharmacometrics, University Children's Hospital Basel (UKBB), University of Basel, Basel, Switzerland; Division of Clinical Pharmacology, Children's National Health Hospital, Washington, DC, USA; and Intensive Care and Department of Pediatric Surgery, Erasmus MC Sophia Children' Hospital, Rotterdam, The Netherlands Lorenz Risch, Private University of the Principality of Liechtenstein, Triesen, Liechtenstein; Labormedizinisches Zentrum Dr. Risch, Vaduz, Liechtenstein; and University Institute of Clinical Chemistry, University of Bern, Bern, Switzerland. https://orcid.org/0000-00032692-6699 